\title{
Management of lower limbs leiomyosarcoma: A case report
}

\author{
Cuenca-González Concepción ${ }^{1}$, Berzal- Cantalejo María Fernanda ${ }^{2}$, Herranz-Torrubiano Ana María $^{3}$ and Lucía Vaamonde Lorenzo ${ }^{4}$ \\ ${ }^{1}$ Universidad Complutense, Faculty of Medicine, Department of Physical Medicine and Rehabilitation, Hospital Clínico San Carlos, Madrid, Spain \\ ${ }^{2}$ Specialist in Anatomical Pathology, Anatomical Pathology Service, Hospital General Río Carrión. Palencia, Spain \\ ${ }^{3}$ Specialist in Family and Community Medicine, Healthcare Centre La Alameda de Osuna, Primary Care, Madrid, Spain \\ ${ }^{4}$ Physical Medicine and Rehabilitation Service, Hospital Clínico San Carlos, Madrid, Spain
}

\begin{abstract}
We report the case of a woman referred to our rehabilitation department who presented a soft tissue tumour, a proximal third tibia leiomyosarcoma. It's analyzed how the diagnosis was made, the treatment for tumour and its sequelae and the follow up period, by doing an update of the scientific evidence in this tumour management.

This tumour is a subtype in soft tissue sarcomas. These are rare tumours that arise mostly from mesenchymal tissues and leiomyosarcoma is the fourth in frequency $(12 \%)$. Although they can appear in any location, $40 \%$ cases have their origin typically in extremities. Sarcomas in extremities are characterized by a painless mass, incidentally discovered by the patient, the doctor or by performing an ultrasound.

Conservative surgery is considered the main treatment for this soft tissue sarcoma, avoiding radical surgeries which don't achieve survival results. Radiotherapy and adjuvant chemotherapy are used both with surgery to prevent local recurrence and metastases.

Pathology and imaging tests (ultrasounds, CT and MRI) will be fundamental for the staging, for therapeutic decisions to be made and for monitoring possible local recurrences or regional or systemic metastases.
\end{abstract}

\section{Case report}

We report the clinical case of a 60 -year-old patient, with no other important medical history but hypertension, dyslipidemia and hypothyroidism with pharmacological treatment, who consulted her family practitioner for a 6 months evolution mass on the lateral aspect of her left leg, lightly painful with palpation, without previous traumatic injury. Ultrasound is requested from Primary care and revealed a solid mass of $66 \times 27 \mathrm{~mm}$ (Figure 1,2).

The patient was sent to her to her reference hospital, to Orthopaedic Surgery service, where the mass was removed. Histological examination showed leiomyosarcoma stage III with surgical margins affectation. (Figure 3)

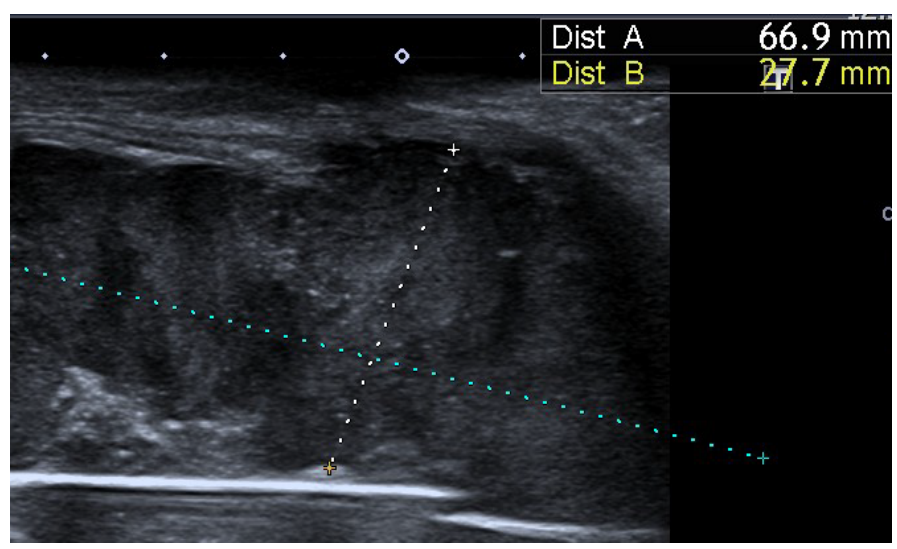

Figure 1. Measurement of solid soft tissue lesion, polylobulated contour. Anterolateral region of the knee

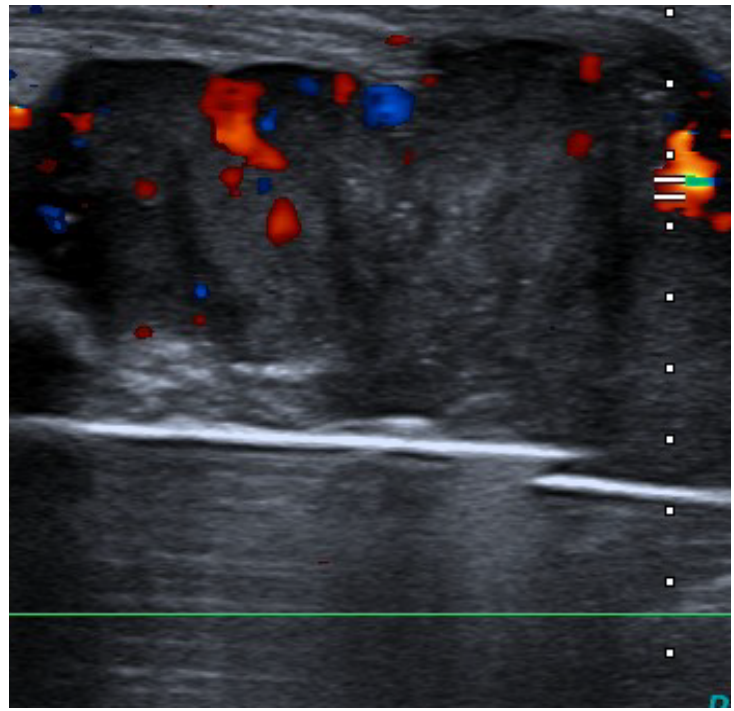

Figure 2. Ultrasound image with Power Doppler where neovascular formation is visualized.

Correspondence to: Cuenca-González Concepción, Universidad Complutense, Faculty of Medicine, Department of Physical Medicine and Rehabilitation, Physical Medicine and Rehabilitation Service, Hospital Clínico San Carlos, Madrid, Spain, Tel: +34 9133036 03; E-mail: cuchacuenca@gmail.com

Key words: soft tissue sarcomas, leiomyosarcoma, conservative treatment of an extremity

Received: November 05, 2017; Accepted: November 06, 2017; Published: November 09, 2017 


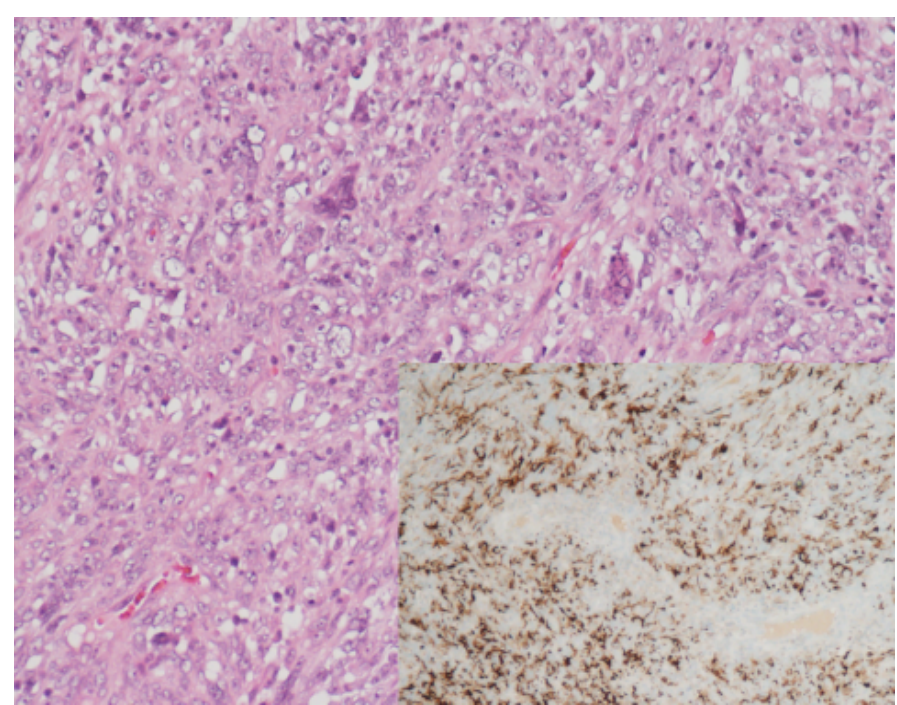

Figure 3. Fascicular proliferation of very atypical cells of mesenchymal nature that show immunohistochemical staining with muscle markers. Hematoxylin-eosin 20X and Desmin 10X

Three months later, an enlargement of surgical margins and skin coverage with medial gastrocnemius flap was performed, also receiving adjuvant radiotherapy. Extension study with thoracoabdominal CT scan and bone scintigraphy were normal, and the MRI didn't detect macroscopic residual tumor.

The patient kept periodical follow up until 5 years later when a MRI reveals a soft tissue solid lesion, located in the upper and outer aspect of the surgical site. This seems to be tumoral recurrence, and it's located nearby patellar tendon that doesn't seem to be affected, also making contact with tibia without subcortical bone invasion (Figure 4, 5).

After multidisciplinary team meeting (Orthopaedic oncology, medical oncology and plastic surgery), it's decided extra-articular resection of the proximal tibia and distal femur, with LINK arthrodesis nail and skin coverage by vascularized free flap from left latissimus dorsi, performed by plastic surgeons (Figure 6).

After satisfactory imaging test and rehabilitation doctor assessment, physical therapy is prescribed during hospitalization, first beginning ambulation with a walker frame three weeks post- surgery, reaching progressive increase of distance up to 10 metres and learning to go up and down stairs. Subsequently, the patient continued physical therapy supervised by her rehabilitation doctor on an outpatient basis.

Two years later, she begins with a chronic infection in her left lower limb with fistulous paths that drain purulent material to the cutaneous surface, periodical cures are needed at the hospital and in primary care centers.

Finally, it's consensually decided with the patient to perform a left transfemoral amputation as a solution to this long chronic infectious process that caused an important functional limitation to her.

The patient is then referred to rehabilitation department again. She doesn't feel phantom limb pain and it's decided to start prosthetization in view of she meet appropriate criteria for this, such as: middle aged, controlled tumour disease, a well-shaped stump without vascular alterations nor alignment problems and painless palpation, getting unipodal stance stability and no other concomitant diseases, among others.

\section{Discussion}

Soft tissue sarcoma (STS) constitute a heterogeneous group of neoplasms of mesenchymal origin, with the exception of tumours of the peripheral nerve, which have ectodermal origin. They constitute $88 \%$ of total sarcomas ${ }^{1}$ and represent $1 \%$ of all malignant tumours of the body. They are usually seen as asymptomatic masses, that's why their diagnosis is delayed, presenting metastasis in $20 \%$ cases when identified, especially in lungs.

Leiomyosarcoma (LMS) is a tumour of smooth muscle cells, thus, it can arise in those locations where there is this type of tissue, such as uterus, gastrointestinal tissue and large vessels [2]. They are classified as uterine, gastrointestinal, retroperitoneal and somatic. Different subtypes of LMS present completely different clinical behaviours and therefore, they should be considered independently $[3,4]$.

Somatic LMS of the soft tissues accounts for approximately 7.6 to $16.1 \%$ of all non-visceral soft tissue sarcomas $[5,6]$. In comparison with other sarcoma subtypes, it represents a relatively aggressive tumor with a high risk of metastasis
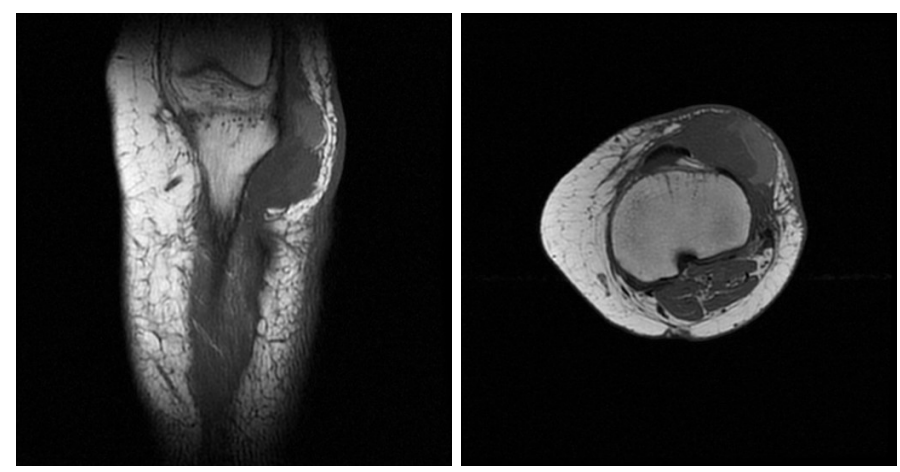

Figure 4, 5. MRI Sagittal and axial sequence T1: Soft tissue lesion, solid, located in the upper and outer aspect of the surgical site, compatible with tumor recurrence, in intimate contact with the patellar tendon without infiltrating it and in contact with the tibia (no subcortical bone invasion), Peripheral portion: Enhancement (bleeding)
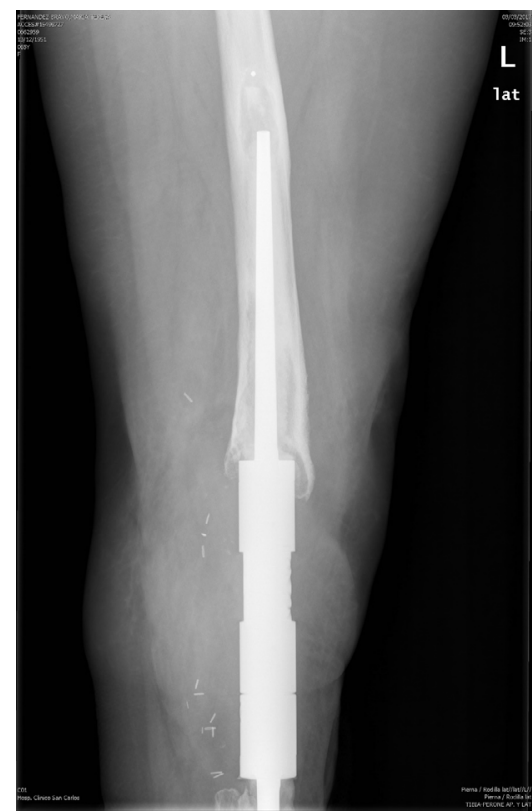

Figure 6. Extra-articular resection of tumor and femoral-tibial arthrodesis using cemented LINK nail 
The average age of STS diagnosis is 50-60 years. Their risk of appearance increases with previous radiotherapy for other neoplasms, ionizing radiation, chronic lymphoedema (lymphangiosarcoma), exposure to vinyl chloride, arsenic, dioxins and Thorotrast (hepatic angiosarcoma). It is also associated with some inherited syndromes (Werner, Gadner, Tuberous sclerosis, Gorlin-Goltz, Von Recklinghausen) [7]. Leiomyosarcomas are not usually associated with radiotherapy but they are associated with complex karyotypes and alterations in the expression of tumor suppressor genes RB1 and TP53. It hasn't been identified genetic or familial predisposition [2]. It has been observed an association between viral infections, such as Epstein Barr virus in patients with AIDS, and leiomyosarcoma [8,9].

More than $50 \%$ of soft tissue sarcomas arise in any of the mesodermal tissues of extremities (only 14\% in upper limbs), being fibrous histiocytoma or pleomorphic sarcoma the most frequent subtype (28\%), followed by liposarcoma (21\%), synovial sarcoma (15\%) and leiomyosarcoma (12\%). The rest are found in trunk and retroperitoneum (40\%) and in head and neck region (10\%) [1].

The most frequent form of presentation is usually a painless, fast-growing tumor of increased consistency. Therefore, Primary Care and Hospital Consultations (Rehabilitation, traumatology and rheumatology) are the most favorable areas for the early detection of these lesions, always keeping in mind the criteria that should make us think of a suspicious tumor. These criteria would be: lesions greater than $5 \mathrm{~cm}$ in size, increased consistency, fast-growing, deep localization and recurrence after surgical removal $[10,11]$. If the patient meet these criteria, the referral to hospital should not be delayed.

The prognosis mainly depends on tumor size, the histological grade and tumor persistence at the surgical margins. High-grade sarcomas are related to higher rates of local treatment failure and higher metastatic potential [12] Table 1

In case of suspicion of STS, first of all, a MRI must be made to assess the extension of the lesion and a biopsy must be done to properly plan surgery [13-15]. The final diagnosis will be made after the histological analysis of the surgical piece. In order to get a curative resection, it is important to plan the initial biopsy, to avoid complications such as those presented by our patient. If high grade STS is confirmed, an extension study should be done with CT to rule out metastases.

A $70 \%$ of distant metastases in patients with extremities sarcomas are usually pulmonary, while in those with sarcoma of the uterus and retroperitoneal tend to be hepatic and pulmonary [2].

The prognosis will depend mainly on the histological grade (most important prognostic factor), histological subtype, tumor size, involvement of surgical margins, localization (better prognosis in extremities), response to treatment and whether it is a primary tumor or a relapse (more unfavorable prognosis) [16,17].

Therapeutic decision will depend on the histology of the tumor, so it is essential for an experienced pathologist to review the biopsied tissue. The treatment is fundamentally based on surgery (complete excision of the lesion with adequate margins) [18] trying to preserve the functionality of the extremity [19], radiotherapy (in unresectable tumors, high probability of residual tumor, margins less than $2 \mathrm{~cm}$, amputation due to extensive resection) [1] and chemotherapy, being the anthracyclines more effective in high-grade tumors (doxorubicin and epirubicin), ifosfamide and dacarbazine [20]. Chemotherapy is used more and more to limit the loss of function after wide surgical excision, with the ultimate goal of improving patient survival [11].
Table 1. AJCC Staging for soft tissue sarcoma. (STS)

AJCC Cancer Staging Manual 7th ed, Springer New York, Usa, 2010

Primitive Tumor (T) TX: Primitive cancer cannot be assesed

T0 there is no evidence of primitive tumor

T1: tumor $<=5 \mathrm{~cm}$ in the larger diameter

T1a: surface tumor (not involving underlying fascia)

T1b: deep tumor (involving or deep to fascia)

T2: tumor $>5 \mathrm{~cm}$ in the larger diameter

T2a: surface tumor

T2b: deep tumor

Regional lymph nodes (N) NX: Regional lymph nodes cannot be assessed

NO: no metastasis in regional lymph nodes

$\mathrm{N} 1$ : metastasis in regional lymph nodes

Distant metastasis (M) M0: No distance metastasis a

M1: Distant metastasis

Histologic grade (G) Gx: Grade cannot be assesed

G1: Grade 1

G2: Grade 2

G 3: Grade 2

Stage T N M G

IA T1a-b N0, NX M0 G1, GX

IB T2 a-b N0, NX M0 G1, GX

IIA T1a -b N0, NX M0 G2, G3

II B $\mathrm{T} 2 \mathrm{a}-\mathrm{b}$ N0, NX M0 G2

III T2a-b N0, NX M0 G3

Any T N1 M0 Any G

IV Any T Any N M1 Any G

In LMS, margins are an important factor in improving local recurrence free survival (LRFS), but none of the retrospective analysis found could establish an association between the quality of the surgical margins and total survival. Therefore, radical surgery in this type of tumor remains controversial [21], since it only seems to have an impact on the local control of the disease.

The oncological outcome seems to be comparable among patients who have undergone a primary or non-primary amputation for extremities soft tissue sarcoma (ESTS) [22]. With the development of the current prosthetization and Rehabilitation programs, remains important to decide, in a multidisciplinary team (oncology, radiology, radiotherapy, rehabilitation, anatomical pathology and surgery) which will be the most appropriate treatment for each patient individually.

\section{Conclusion}

Sarcomas are rare tumors that we should suspect in our Primary Care and Hospital Care consultations in presence of any soft tissue mass of suspicious characteristics and mainly located in lower extremities. A misdiagnosis or a delay in its realization can suppose an important deterioration in quality of life and survival of these patients. A multidisciplinary team for the management of these patients it's needed, and it is also necessary to assess each case individually, trying to perform limbs conservative surgery because no better survival results were obtained with radical surgery.

\section{Funding}

There are no sources of funding or financial support.

\section{Disclosures}

The authors have no conflicts of interest or financial ties to disclose. 


\section{References}

1. Villalobos León ML (2013) Sarcoma de partes blandas. Medicine 11: 1659-1668.

2. Matushsnsky I, Henseley M (2006) Leiomyosarcoma: An Overview of Etiology, prognosis and treatment Options. American Journal of cáncer 5: 81.91.

3. Miettinen M, Fetsch JF (2006) Evaluation of biological potential of smooth muscle tumours. Histopathology 48: 97-105. [Crossref]

4. Pijpe J, Broers GH, Plaat BE, Hundeiker M, Otto F, et al. (2002) The relation between histological, tumor-biological and clinical parameters in deep and superficial leiomyosarcoma and leiomyoma. Sarcoma 6:105-110. [Crossref]

5. Hung GY, Yen CC, Horng JL, Liu CY, Chen WM, et al. (2015) Incidences of Primary Soft Tissue Sarcoma Diagnosed on Extremities and Trunk Wall: A Population-Based Study in Taiwan. Medicine (Baltimore) 94: e1696. [Crossref]

6. Mastrangelo G, Coindre JM, Ducimetière F, Dei Tos AP, Fadda E, et al. (2012) Incidence of soft tissue sarcoma and beyond: a population-based prospective study in 3 European regions. Cancer 118: 5339-5348. [Crossref]

7. Miguel Sepulveda H (2004) Clínica y Generalidades de Sarcoma de partes blandas. Medwave 2004 Abr, 4: e3284 doi: 10.5867/medwave.2004.03.3284.

8. Smith HG, Memos N, Thomas JM, Smith MJ, Strauss DC, et al. (2016) Patterns of disease relapse in primary extremity soft-tissue sarcoma. Br J Surg 103: 1487-1496. [Crossref]

9. Mastrangelo G, Coindre JM, Ducimetiere F, Dei Tos AP Fadda E, et al. (2012) Incidence of soft tissue sarcoma and beyond: a population-based prospective study in 3 European regions. Cancer 118: 5339-5348. [Crossref]

10. Sinha S, Peach AH (2010) Diagnosis and management of soft tissue sarcoma. BMJ 341: c7170. [Crossref]

11. Guerrini GP, Bertola G (2017) Soft Tissue Sarcomas of the Limbs: Multidisciplinarytreatment. WCRJ 4: e 917
12. Singer S, Maki RG, O’Sullivan B (2011) Soft Tissue sarcoma. In: De vita VT Jr, Lawrence TS, Rosemberg SA: Cancer: Principles and Practice of Oncology. 9th ed. Philadelphia, Pa: Lippicott Wliiams \&Wilkins, pp 1533-1577.

13. Shapeero LG, Vanel D, Verstraete KL, Bloem JL (2002) Fast magnetic resonance imaging with contrast for soft tissue sarcoma viability. Clin Orthop Relat Res 397: 212-227.

14. Chan WP (2013) Magnetic resonance imaging of soft-tissue tumors of the extremities: A practical approach. World J Radiol 5: 455-459. [Crossref]

15. De La Hoz Polo, Dick E, Bhumbra R, Pollock R, Sandhu R, et al. (2017) Surgical considerations when reporting MRI studies of soft tissue sarcoma of the limbs. Skeletal Radiol 46:1667-1678

16. Fiore M, Casali PG, Miceli R, Mariani L, Bertulli R, et al. (2006) Prognostic effect of re-excision in adult soft tissue sarcoma of the extremity. Ann Surg Oncol 13: 110-117. [Crossref]

17. Ipach I, Wingert T, Kunze B, Kluba T (2012) Oncological outcome and prognostic factors in the therapy of soft tissue sarcoma of the extremities. Orthop Rev (Pavia) 4: e34. [Crossref]

18. Stojadinovic A, Leung D, Hoos A, Jaques DP, Lewis JJ, et al. (2002) Analysis of the prognostic significance of microscopic margins in 2084 localized primary adult soft tissue sarcomas. Ann Surg 235:424-434. [Crossref]

19. Katz SC (2017) Tailoring Surgical Therapy for Extremity Soft Tissue Sarcoma. Ann Surg Oncol 24: 13-14. [Crossref]

20. Patrikidou A, Domont J, Cioffi A, Le Cesne A (2011) Treating soft tissue sarcomas with adjuvant chemotherapy. Curr Treat Options Oncol 12: 21-31. [Crossref]

21. Harati K, Daigeler A, Lange K, Niggemann H, Stricker I, et al. (2017) Somatic Leiomyosarcoma of the Soft Tissues: A Single-Institutional Analysis of Factors Predictive of Survival in 164 Patients. World J Surg 41:1534-1541. [Crossref]

22. Stevenson MG, Musters AH, Geertzen JHB, van Leeuwen BL, Hoekstra HJ, et al. (2017) Amputations for extremity soft tissue sarcoma in an era of limb salvage treatment: Local control and survival. J Surg Oncol 1-9. [Crossref]

Copyright: (C)2017 Concepción CG. This is an open-access article distributed under the terms of the Creative Commons Attribution License, which permits unrestricted use, distribution, and reproduction in any medium, provided the original author and source are credited. 